\title{
INFLUÊNCIA DE 15 ANOS DE SOBRECARGA DE A- TERRO NA CARACTERIZAÇÃO GEOTÉCNICA DE UM DEPÓSITO DE ARGILA MOLE
}

\author{
Bianca da Silva Baldez ${ }^{1}$ \\ Denise Maria Soares Gerscovich ${ }^{2}$ \\ Bernadete Ragoni Danziger ${ }^{3}$ \\ Rachel da Silva Azevedo ${ }^{4}$
}

\begin{abstract}
Resumo - A previsão do comportamento de aterros em solos argilosos de baixa consistência representa um desafio, mesmo para engenheiros geotécnicos mais experientes. Depósitos argilosos moles ocorrem em áreas de baixada e são frequentes nas zonas costeiras brasileiras. Para a avaliação da compressibilidade destes depósitos recomenda-se, em geral, a realização de ensaios de adensamento convencional ou com velocidade controlada. $\mathrm{O}$ presente artigo tem como objetivo apresentar os resultados de duas campanhas de ensaios de laboratório; uma realizada em 1997 e outra em 2012, em um mesmo depósito de argila mole, localizado na Baixada de Jacarepaguá, Rio de Janeiro. A campanha de 1997 foi feita na fase do projeto de implantação de um Centro Comercial de Serviços, o qual envolveu a execução de extenso aterro. As investigações de 2012 objetivaram avaliar a influência de 15 anos de atuação da sobrecarga do aterro, nos parâmetros de compressibilidade do depósito. Os resultados mostraram que a qualidade dos corpos de prova da primeira campanha foram superiores aos da campanha mais atual, apesar dos cuidados com a amostragem, transporte e preparação dos corpos de prova no laboratório na campanha mais recente. Adicionalmente, foram observados que os critérios de qualidade sugeridos na literatura são extremamente rigorosos para o caso das amostras ensaiadas. As principais diferenças na caracterização geotécnica foram (i) redução significativa do índice de vazios; (ii) valores de OCR tendendo a 1 e (iii) redução de cerca de $65 \%$ no índice de compressibilidade $\mathrm{C}_{\mathrm{c}}$.
\end{abstract}

Palavras-chave: compressibilidade, ensaio de adensamento convencional; ensaio de adensamento com velocidade controlada.

Abstract - The prediction of the behavior of an extensive sandy embankment on a soft clay foundation is a challenge task, even for geotechnical engineers with great expertise. Clayey deposits occur in lowlands, being very common in Brazilian coast. In order to evaluate the compressibility characteristics of such deposits, standard incremental consolidation and constant rate of strain tests are usually performed. The aim of the present paper is to present the results of two geotechnical campaign performed on samples obtained from a very soft clay deposit from Jacarepaguá, in Rio de Janeiro. The second campaign has been performed 15 years interval after the end of the fill construction. The new samples have been collected very close to the boring verticals of the first campaign. The new geotechnical investigation was able to evaluate the clay characteristics and compressibility after the action of the fill surcharge. The results illustrated that the sample quality were superior in the first campaign although the recent sample extraction, transport and manipulation in the laboratory have been conducted with extreme caution. Additionally, the quality criteria suggested in the literature has been observed as being extremely rigorous for the samples analyzed in the present article. The main differences observed in the recent characterization of the clay deposit were: significant reduction in void ratio; (ii) OCR values tendency to approach unity and (iii) reduction in soil compressibility by nearly $65 \%$.

\footnotetext{
${ }^{1}$ Universidade Estadual do Rio de Janeiro. E-mail: bbvbaldez@gmail.com

${ }^{2}$ Universidade Estadual do Rio de Janeiro. E-mail: deniseg@uerj.br

${ }^{3}$ Universidade Estadual do Rio de Janeiro. E-mail: bernadeterd@ hotmail.com

${ }^{4}$ Universidade Estadual do Rio de Janeiro. E-mail: rachel.azevedo@live.com
} 
Key words: compressibility, standard consolidation test; CRS consolidation test.

\section{INTRODUÇÃO}

As áreas litorâneas costumam apresentar uma ocupação urbana intensa, face à proximidade com o mar. Nessas regiões encontram-se os depósitos argilosos, superficiais, muito moles a moles, de idade geológica recente e de espessura variável.

A cidade do Rio de Janeiro tem crescido em direção à Zona Oeste da Cidade, aqui denominada de Baixada de Jacarepaguá, onde se localiza o extenso depósito de solo compressível, objeto deste trabalho.

Depósitos moles resultam do acúmulo de minerais, de granulometria fina, em vários tipos de ambientes sedimentares, podendo apresentar certo teor de matéria orgânica proveniente da decomposição de restos de animais e vegetais. Quando o teor de matéria orgânica é predominante são denominados de solos orgânicos ou turfas.

Segundo Sandroni (1980), podem ser reconhecidos diversos ambientes que influenciam o tipo de depósito a ser formado, tais como os fluviais (leitos de rios abandonados), deltaicos-fluviais (rios ou lagos, local de águas calmas) e costeiros (influência da flutuação da maré). Os sedimentos que constituem estes depósitos moles são de origem geológica recente correspondente ao período holoceno.

Os grãos minerais que constituem de forma predominante estes depósitos estão relacionados à forma e ao meio de deposição. No Brasil o mineral argílico mais frequente é a caulinita, sendo também possível a presença de ilita, haloisita, montmorilonita e clorita. A composição mineralógica define as características das partículas de argila, tais como dimensão, forma e superfície. A fração de argila influencia no comportamento do solo quanto à plasticidade, potencial de expansão e compressibilidade. As argilas moles brasileiras apresentam geralmente processos semelhantes de deposição em regiões costeiras, como os depósitos de Sarapuí na Baixada
Fluminense, da Baixada Santista e de Pernambuco.

Este trabalho tem como objetivo apresentar os resultados de campanhas de ensaios de laboratório, realizadas em 1997 e 2012, em amostras de argila mole de um depósito da Baixada de Jacarepaguá. A primeira campanha precedeu à implantação de um empreendimento comercial no local, o qual exigiu o lançamento de um extenso aterro. Já a segunda campanha irá avaliar as condições atuais do depósito, após 15 anos de sobrecarga de aterro.

\section{DESCRIÇÃO DO LOCAL}

O local em estudo refere-se a um Centro Comercial de Serviços, com cerca de $30.000 \mathrm{~m}^{2}$ de área, situado à Av. Ayrton Senna, Barra da Tijuca. O empreendimento, construído em 1997, engloba edificações, assentes sobre estacas metálicas, e uma área aterrada para fins de estacionamento. A Figura 1 mostra a vista atual do local; à esquerda o terreno permanece intacto e à direita foi construída outra edificação comercial com subsolo.

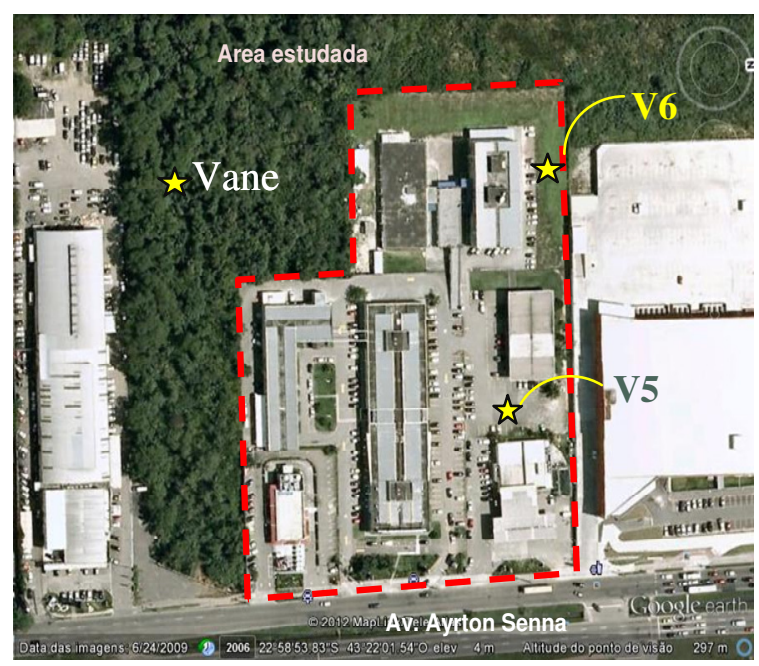

Figura 1- Vista do empreendimento e localização de parte dos ensaios de palheta. 
O projeto previa um aterro de areia média limpa, com cerca de $1,7 \mathrm{~m}$ de altura. Entre o aterro e solo natural foi instalada uma manta de geotêxtil (Figura 2).

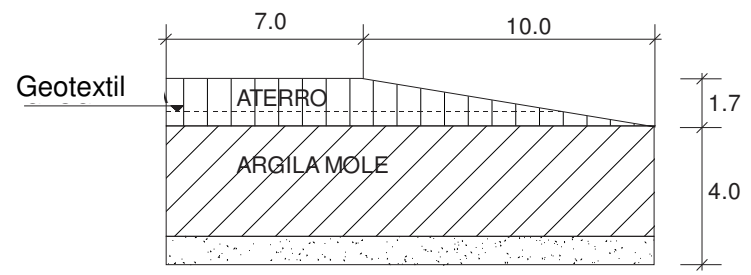

Figura 2- Projeto do aterro (Baldez, 2013).

$\mathrm{Na}$ fase de projeto, as investigações envolveram a execução de sondagens a percussão, de ensaios de palheta (Vane) em seis verticais, sendo que próximo às verticais (V5 e V6), indicadas na Figura 1, foram retiradas amostras indeformadas para ensaios de laboratório. No laboratório, foram realizados ensaios de adensamento convencionais (SIC) em amostras extraídas próximas aos perfis de palheta.

A Erro! Fonte de referência não encontrada. mostra um perfil típico onde se observa que os primeiros 3 a 4 metros são constituídos por camadas de solos moles sobrejacentes a um solo arenoso. Maiores detalhes sobre o posicionamento das investigações podem ser encontrados em Baldez (2013).

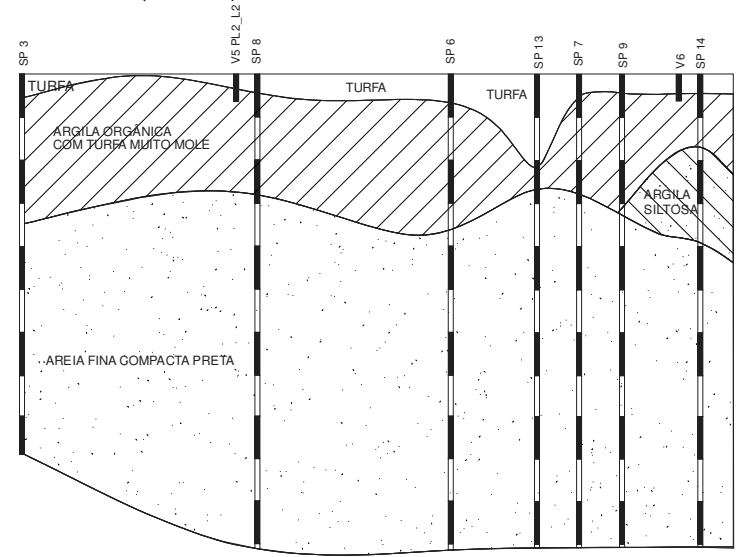

Figura 3- Perfil geotécnico (Baldez, 2013).
Para os trechos de cerca de $4,0 \mathrm{~m}$ de espessura de camada mole foram previstos recalques por adensamento primário da ordem de 1,20 m, considerando o efeito da submersão do aterro.

\section{CARACTERIZAÇÃO GEOTÉCNICA EM 1997}

As curvas granulométricas (Figura ) mostram uma tendência de redução da porcentagem de argila com a profundidade (V5), sendo a porcentagem de argila em V6 inferior à vertical V5, além da presença de um veio arenoso (amostra V6-3).

Os ensaios de caracterização (Figura ) registraram um material com umidade de cerca de $500 \%$ na superfície até $1,00 \mathrm{~m}$ de profundidade, reduzindo para $200 \%$ de 1,00 a $3,00 \mathrm{~m}$, e voltando a valores elevados nas profundidades de 3,00 a 4,00 m. Os índices de vazios apresentaram valores extremamente elevados, variando de 6 a 13. O peso específico natural, da ordem de $12 \mathrm{kN} / \mathrm{m}^{3}$, foi considerado baixo em relação a outros depósitos de argila mole já documentados. Adicionalmente, os valores de limite de liquidez variaram de 460 a $796 \%$ e limite de plasticidade de 103 a $181 \%$. 


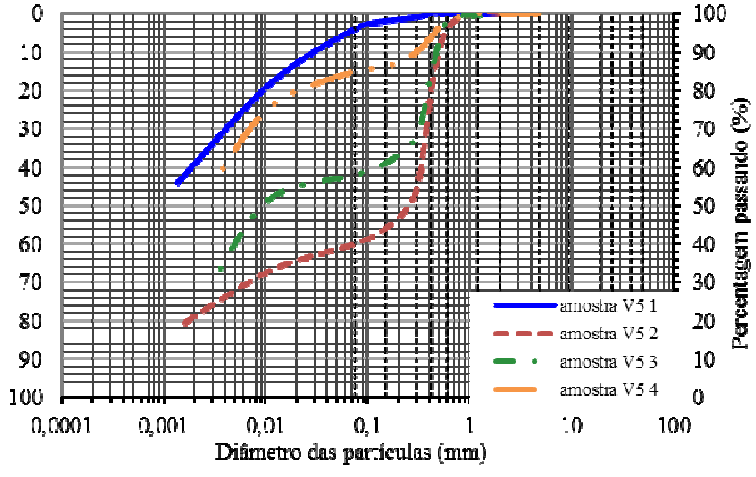

(a) Vertical V5

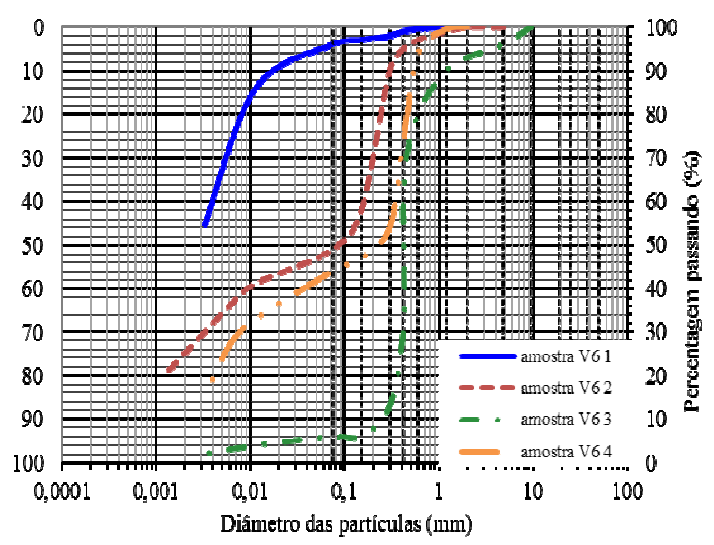

(b) Vertical V6

Figura 4 - Curvas granulométricas verticais V5 e V6 (ensaios 1997).

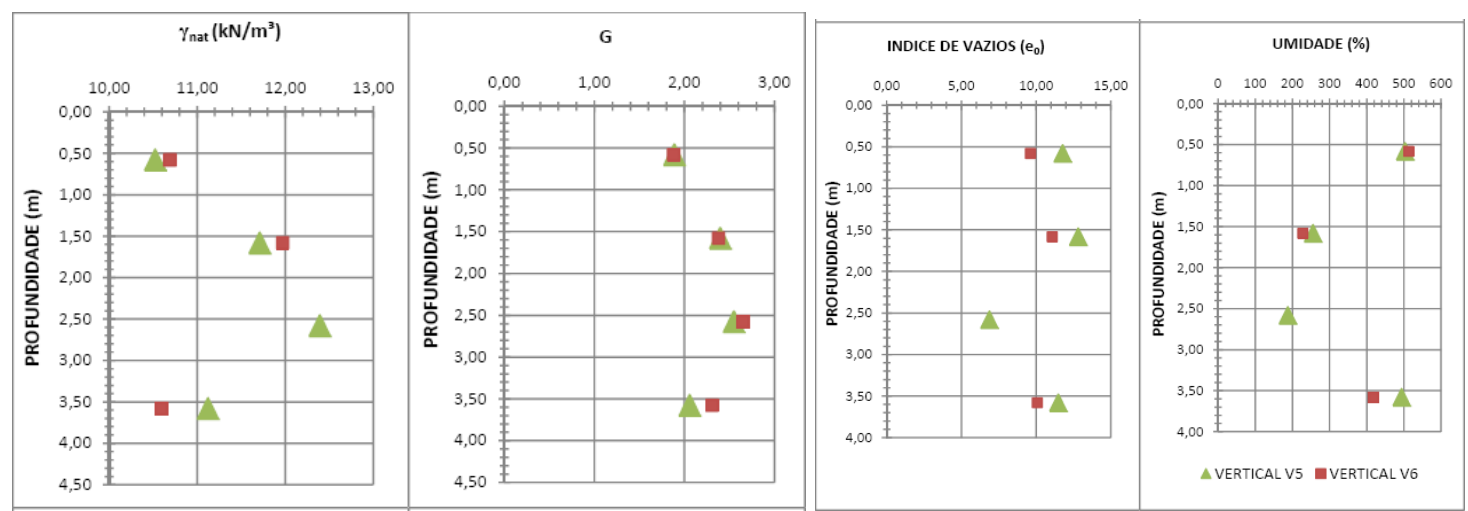

Figura 5 - Parâmetros geotécnicos, ensaios de 1997.

Cabe destacar a similaridade dos resultados dos ensaios de caracterização com outros casos de obra, referentes à Baixada de Jacarepaguá, obtidos por Bedeschi (2004), Spotti (2000 e 2006), Sandroni (2012).

Com base nas curvas de compressibilidade (

Figura ) foi realizado estudo sobre a confiabilidade dos resultados experimentais, visto que na época do projeto não havia critério para aferir a qualidade das amostras. Segundo o critério sugerido por
Lunne et al. (1997), observou-se (Tabela 1), que a maioria dos corpos de prova era de boa qualidade. Cabe ressaltar que o critério de Lunne et al (1997) foi estabelecido para solos com OCRs inferiores a 4 e que esta limitação foi desconsiderada tendo em vista que a forma da curva mostrava claros indícios de ser um corpo de prova de boa qualidade: (i) não achatamento da curva; (ii) não linearidade do trecho virgem. 


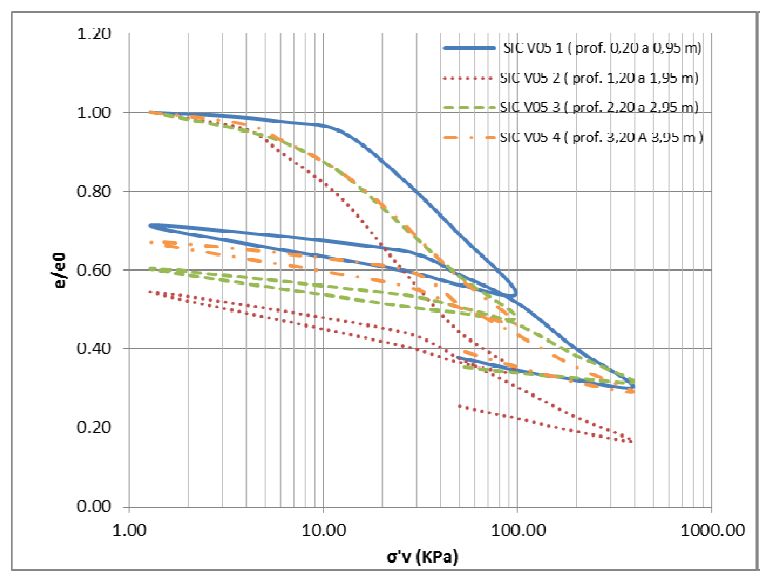

(a) Posição próxima à V5

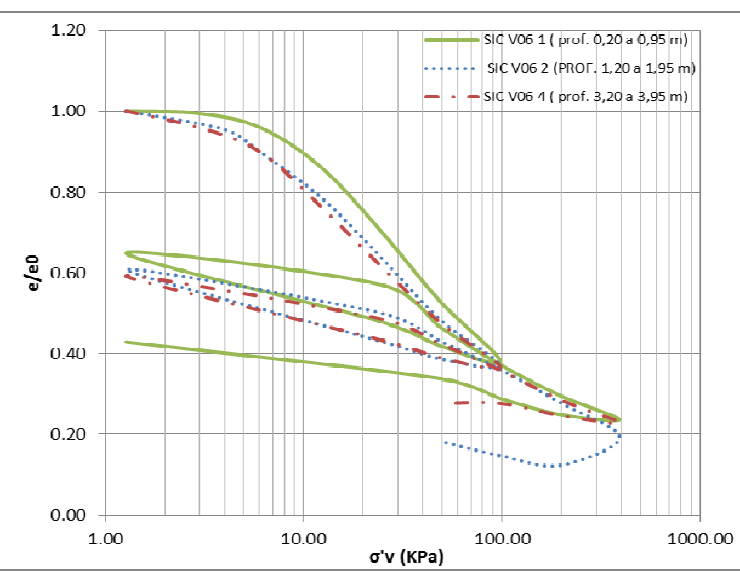

(b) Posição próxima à V6

Figura 6- Curvas de compressibilidade (ensaios 1997).

Tabela 1 - Qualidade dos corpos de prova (ensaios 1997).

\begin{tabular}{c|c|c|c|c|c|c|c}
\hline Posição & Amostra & $\begin{array}{c}\sigma^{\prime}{ }_{\mathrm{vm}} \\
(\mathrm{kPa})\end{array}$ & $\begin{array}{c}\sigma_{\mathrm{v} 0}^{\prime} \\
(\mathrm{kPa})\end{array}$ & OCR & $\mathrm{e}_{0}$ & $\Delta \mathrm{e}^{\prime} \mathrm{e}_{0}$ & Classificação \\
\hline \multirow{4}{*}{$\begin{array}{c}\text { Vertical } \\
\text { V5 }\end{array}$} & 1 & 15 & 0,30 & 50,0 & 11,8 & 0,01 & Excelente a Muito Boa \\
\cline { 2 - 8 } & 3 & 7 & 1,51 & 4,6 & 12,8 & 0,02 & Excelente a Muito Boa \\
\cline { 2 - 8 } & 4 & 9 & 5,27 & 1,7 & 11,5 & 0,05 & Boa a Aceitável \\
\hline \multirow{4}{*}{$\begin{array}{c}\text { Vertical } \\
\text { V6 }\end{array}$} & 1 & 9 & 0,30 & 30,0 & 9,6 & 0,01 & Excelente a Muito Boa \\
\cline { 2 - 8 } & 3 & 7 & 1,51 & 4,6 & 11,1 & 0,02 & Excelente a Muito Boa \\
\cline { 2 - 8 } & 4 & - & - & - & - & - & Veio Solo Arenoso \\
\hline
\end{tabular}

A Figura resume os parâmetros de compressibilidade e adensamento. Observa-se que, em face de tensões de préadensamento na faixa de 6 a $10 \mathrm{kPa}$, identificou-se uma crosta superficial com OCR superior a 4 e uma tendência de $\mathrm{OCR}=1$ na base da camada mole.

O coeficiente de adensamento médio na vertical V5 está na faixa próxima a $1 \times 10^{-4} \mathrm{~cm}^{2} / \mathrm{seg}$. Já na vertical V6, a dispersão foi menor e o $\mathrm{c}_{\mathrm{v}}$ médio foi de $2 \times 10^{-}$ ${ }^{5} \mathrm{~cm}^{2} / \mathrm{seg}$. Curiosamente, na vertical V6 foi observada uma maior porcentagem de areia e, neste caso, seriam esperados maiores valores de $\mathrm{c}_{\mathrm{v}}$. Com isso, os autores atribuem este comportamento atípico à dispersão natural no valor de $c_{v}$ e não a um determinado padrão de comportamento

Os valores médios dos coeficientes de compressibilidade $\mathrm{Cc}$ e $\mathrm{C}_{\mathrm{r}}$ foram da ordem de 5 e 1 , respectivamente, e não se observou redução na dispersão dos resultados quando os valores foram normalizados por $\left(1+\mathrm{e}_{\mathrm{o}}\right)$, como mostra a Tabela 2. 


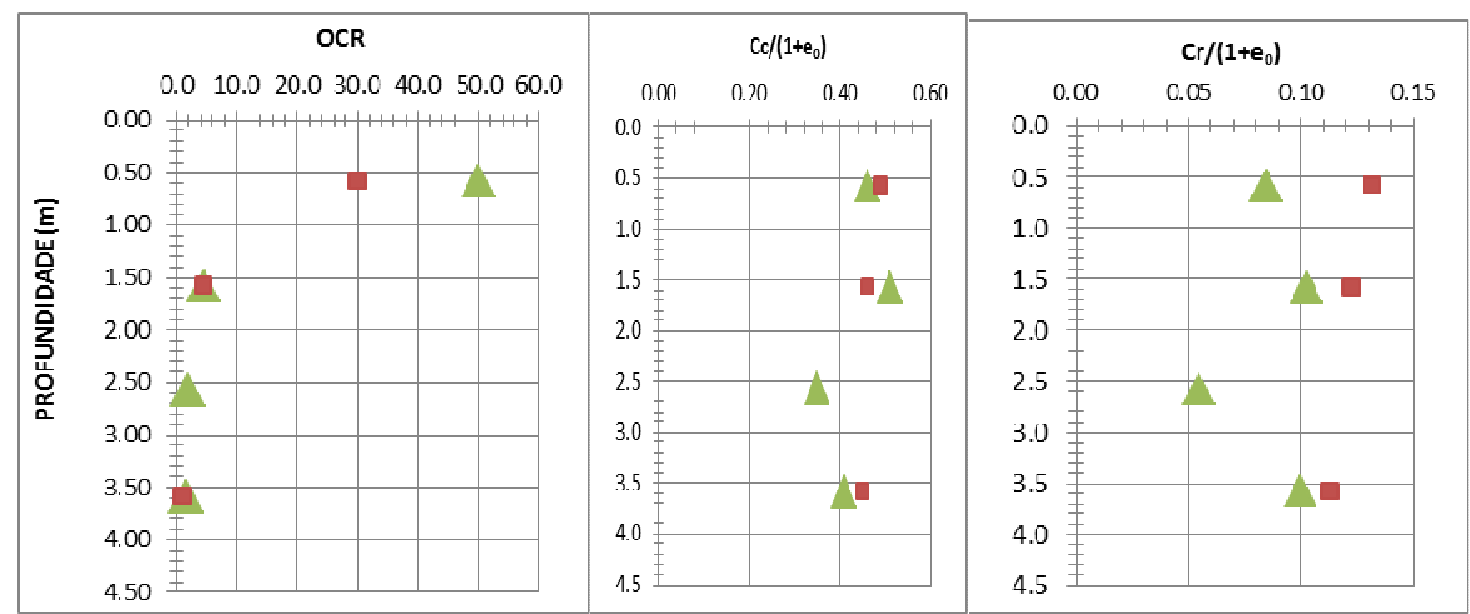

(a) Coeficientes de Compressibilidade

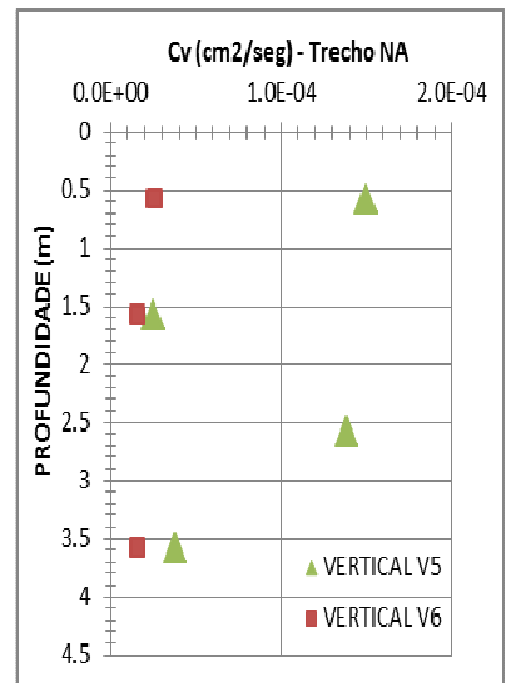

(b) Coeficiente de adensamento

Figura 7 - Parâmetros de compressibilidade e adensamento (ensaios 1997).

Tabela 2 - Parâmetros de compressibilidade normalizados.

\begin{tabular}{c|c|c}
\hline AMOSTRA & $\mathrm{Cc} /\left(1+\mathrm{e}_{0}\right)$ & $\mathrm{C}_{\mathrm{r}} /\left(1+\mathrm{e}_{0}\right)$ \\
\hline V5 01 & 0,46 & 0,03 \\
\hline V5 02 & 0,51 & 0,08 \\
\hline V5 03 & 0,35 & 0,05 \\
\hline V5 04 & 0,41 & 0,10 \\
\hline V6 01 & 0,49 & 0,13 \\
\hline V6 02 & 0,46 & 0,12 \\
\hline V6 03 & - & - \\
\hline V6 04 & 0,45 & 0,11 \\
\hline
\end{tabular}

\section{CARACTERIZAÇÃO} GEOTÉCNICA DO SUBSOLO EM 2012

A amostragem foi feita no inicio do mês de dezembro de 2012, com a retirada de amostras shelby em verticais próximas aos locais onde foram realizados os ensaios de palheta V5 e V6 (Figura 1).

As sondagens respeitaram a norma NBR 9820/1997 referente à retirada de amostras indeformadas em solos moles. $\mathrm{O}$ amostrador utilizado foi o Osterberg, indicado para argilas orgânicas moles, solos argilosos e areias. Vale ressaltar que durante o tempo esperado para recuperação das amostras, o peso do amostrador foi mantido sobre o tubo Shelby, diferentemente do que recomenda a norma.

Durante os trabalhos de campo observou-se a presença de areia limpa, pro- 
veniente do aterro, seguida de turfa (Figura 4) além de conchas (Figura 5).

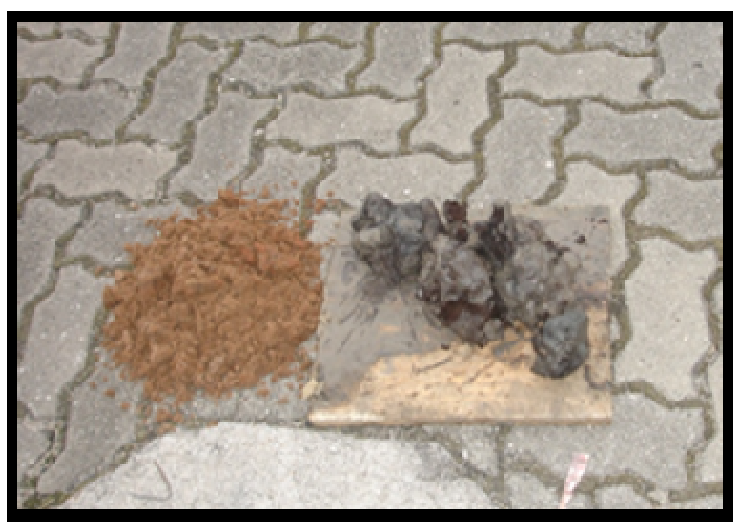

Figura 4- Presença de areia seguida de turfa na região superficial.

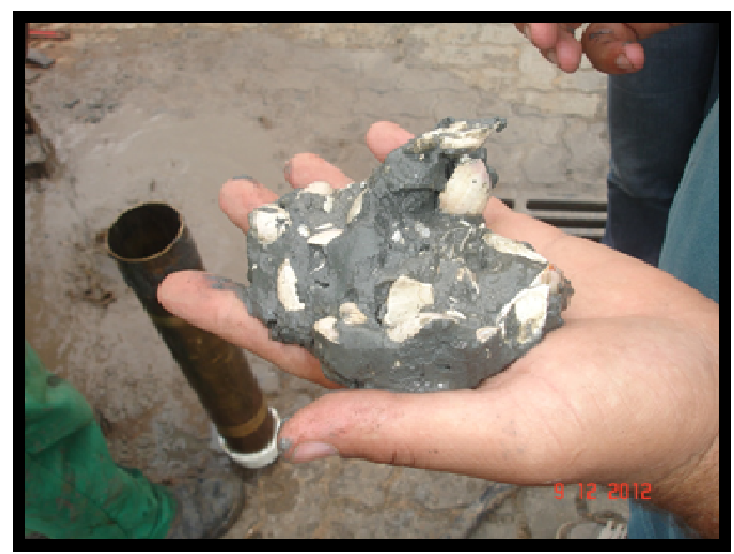

Figura 5- Presença de conchas.

Durante a operação de preparação dos corpos de prova, foram observadas algumas dificuldades do tipo: (i) Presença de pedaços de madeira (Figura 10); (ii) grande quantidade de areia (Figura 7); iii) Fissuras na amostra (Figura 12); iv) Geotêxtil (Figura 9).

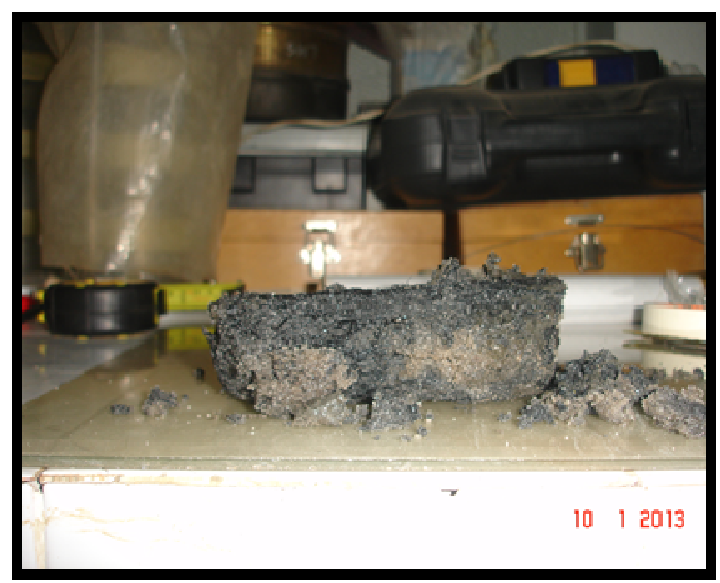

Figura 6- Presença de madeira.

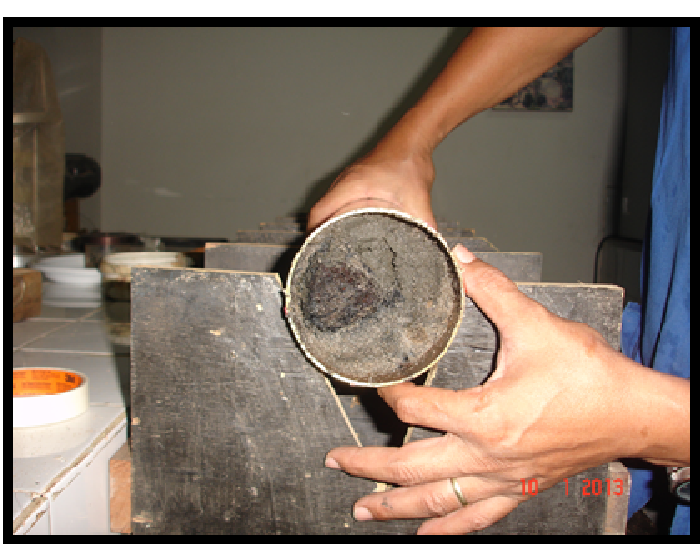

Figura 7- Presença de areia.

Ensaios de caracterização foram realizados seguindo as determinações da Norma Brasileira, com exceção da determinação dos limites de consistência que foi feita sem secagem prévia.

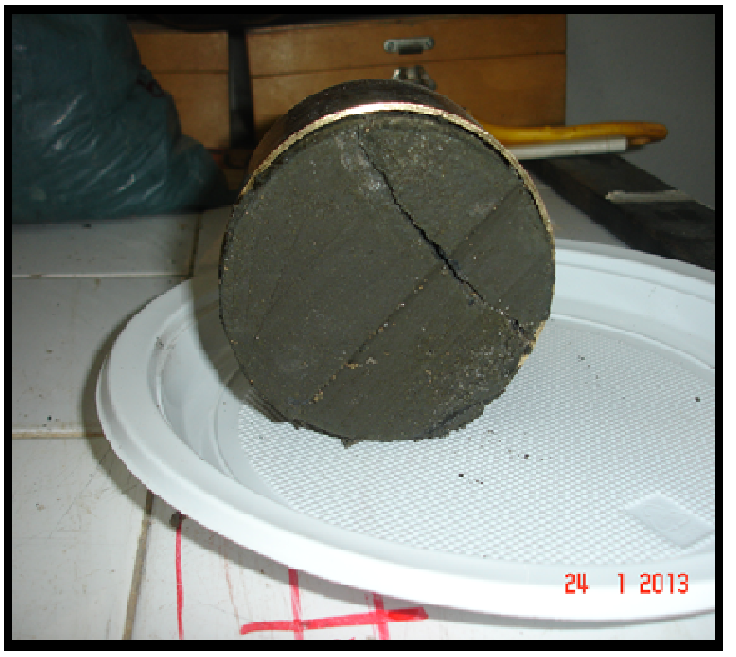

Figura 8 - Fissura na amostra.

As curvas granulométricas (Figura 10) mantiveram a mesma tendência. Curiosamente observou-se redução significativa da \%argila nas amostras realizadas. Quanto à caracterização (Figura 14 e Tabela 3), observa-se o material mais superficial com maior teor de argila. 


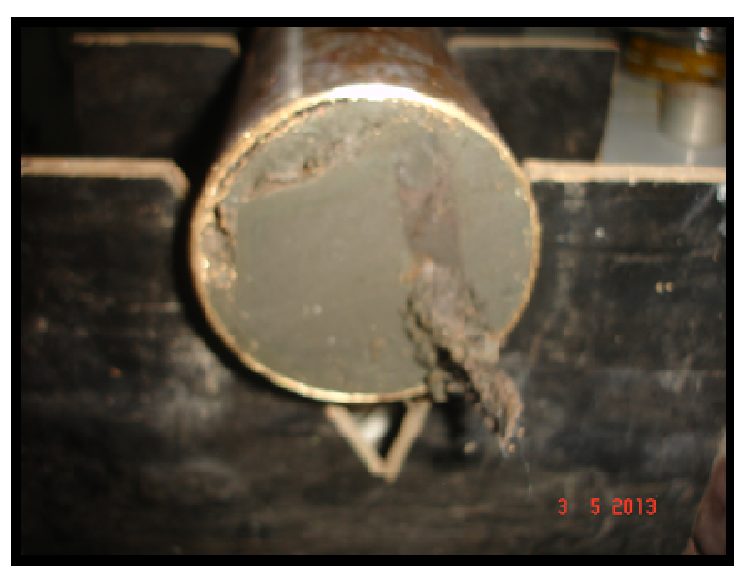

Figura 9 - Tira de geotêxtil.

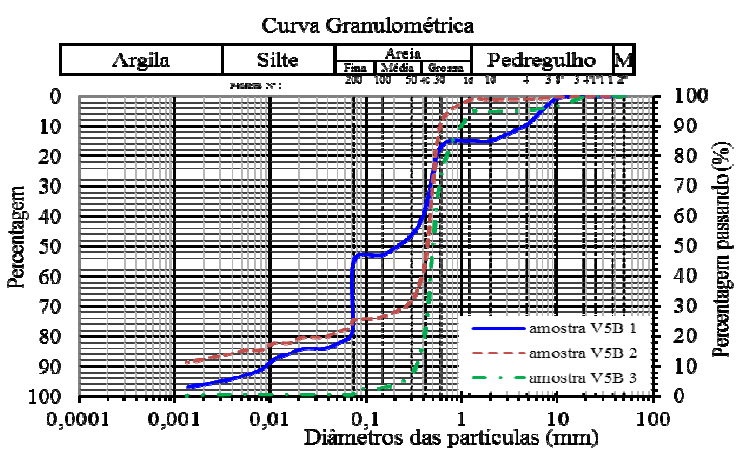

(a) Vertical V5
Baroni (2010), em análise de argilas orgânicas muito compressíveis da Barra da Tijuca apresentou valores de LP variando de $250 \%$ a $111 \%$, e LL de $41 \%$ a $71 \%$, como característica típica de solos moles costeiros da região Sudeste do Brasil.

Figura 10 - Curvas granulométricas - ensaios 2012.

Tabela 3 - Ensaios de caracterização - ensaios 2012.

\begin{tabular}{c|c|c|c|c|c|c|c|c}
\hline \multirow{2}{*}{ Amostra } & Prof. & $\gamma$ nat & \multirow{2}{*}{$\mathrm{G}$} & \multirow{2}{*}{$\mathrm{e}_{0}$} & $\mathrm{w}_{\text {nat }}$ & $\mathrm{LL}$ & $\mathrm{LP}$ & $\mathrm{IP}$ \\
\cline { 2 - 5 } \cline { 6 - 9 } & $(\mathrm{m})$ & $(\mathrm{KN} / \mathrm{m} 3)$ & & & $(\%)$ & $(\%)$ & $(\%)$ & $(\%)$ \\
\hline V5B 1 & 2,87 & 13,80 & 2,56 & 2,54 & 90,38 & 218,79 & 65,78 & 153,00 \\
\hline V5B2 & 3,80 & 13,20 & 2,60 & 3,52 & 130,15 & 45,00 & 16,41 & 28,59 \\
\hline V6A 1 & 2,29 & 12,80 & 2,53 & 3,68 & 136,74 & 102,80 & 34,20 & 68,60 \\
\hline
\end{tabular}

Os parâmetros de compressibilidade e de adensamento foram determinados em ensaios convencionais (SIC) e de deformação controlada (CRS), em amostras extraídas do mesmo local das obtidas em 1997.

Os ensaios CRS foram executados no equipamento automatizado GeoStar S5211, mostrado na Figura 11. A condição não drenada é imposta na base da célula e a geração de poro pressão é acompanhada por transdutor de pressão; uma célula de carga posicionada no topo registra as cargas verticais aplicadas ao corpo de prova. Maiores detalhes sobre o



(b) Vertical V6 


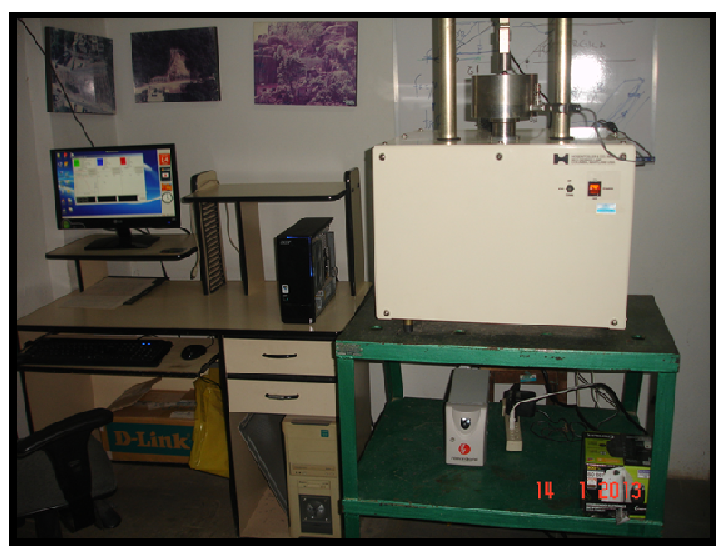

A Figura 12 mostra as curvas de compressibilidade obtidas nos diferentes ensaios. No caso do ensaio CRS, estão apresentadas curvas com diferentes velocidades de deformação. Os ensaios CRS01, CRS02, CRS06, CRS07, CRS09 e CRS10 foram descartados, devido aos problemas ocorridos e detalhados por Baldez (2012).

Figura 11 - Equipamento para ensaio CRS.

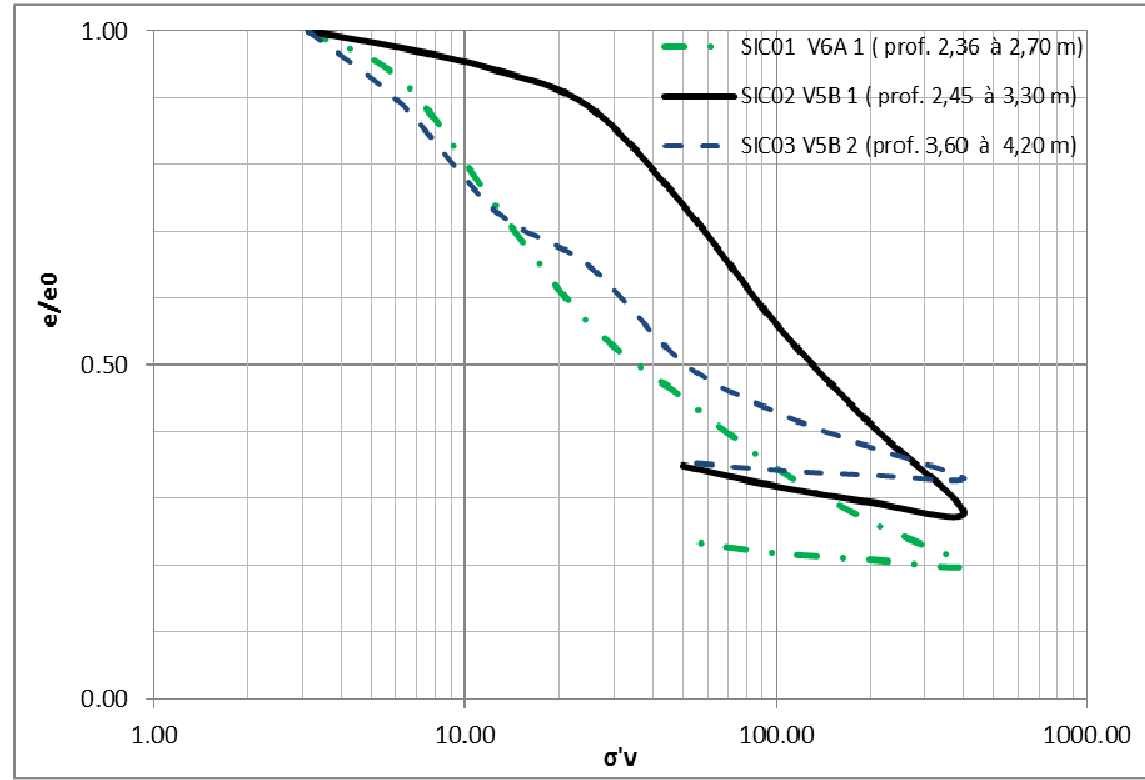

(a) Ensaio SIC

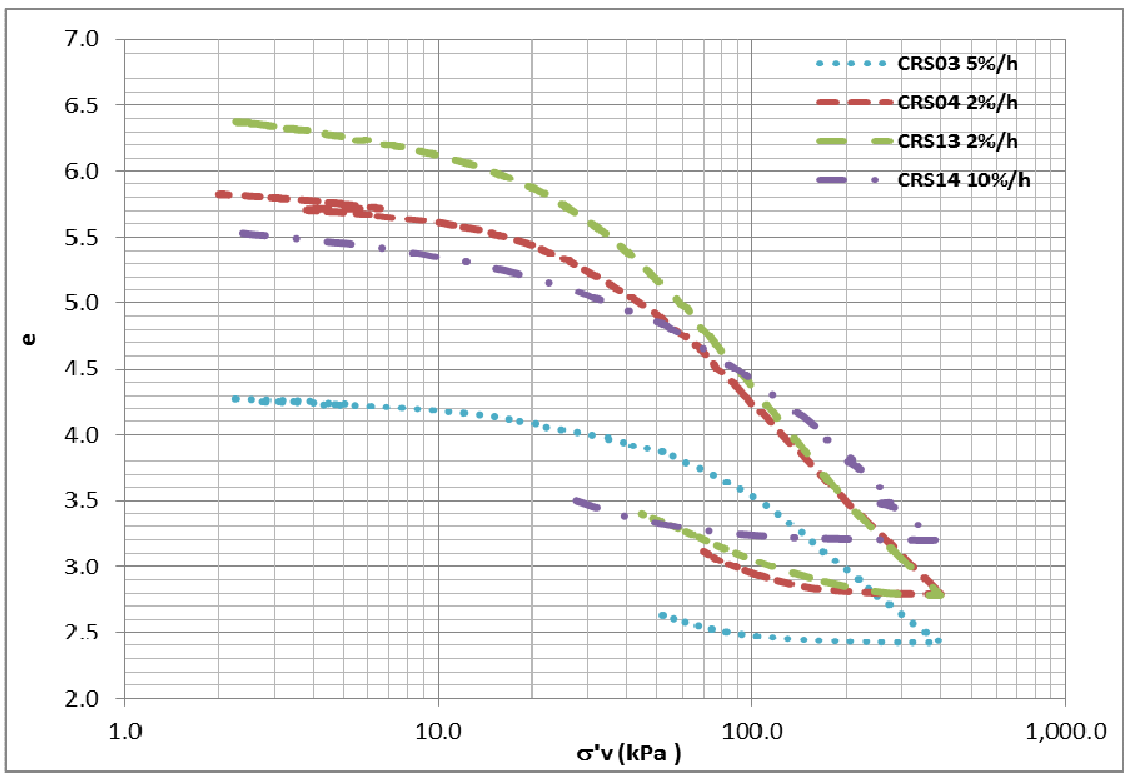

(b) Ensaio CRS

Figura 12 - Curvas de compressibilidade (ensaios 2012) 
O ensaio CRS com velocidade mais elevada $(10 \%)$ resultou em comportamento semelhante ao produzido pelo amolgamento; isto é, índice de recompressão $\left(\mathrm{C}_{\mathrm{r}}\right)$ mais elevado.

A verificação da qualidade dos corpos de prova foi feita segundo os critérios de Lunne et al. (1997), Coutinho (2007) e Andrade (2009). No caso dos ensaios convencionais as amostras apresentaram valores de $\Delta \mathrm{e}^{\mathrm{e}} \mathrm{e}_{0}$ variando de 0,17 a 0,41 , tendo sido classificadas, em todos os critérios, como "muito ruins". Como resultado, os ensaios SIC foram descartados. Já no caso dos ensaios CRS (Tabela 4), a classificação variou entre boa e ruim.

Apesar da qualidade da amostra CRS 03 ter sido considerada adequada, a geração da poropressão na base $\left(\mathrm{u}_{\mathrm{b}}\right)$ foi sempre negativa, indicando problema de saturação da linha que leva ao transdutor ou de vedação do equipamento. Cabe ob- servar, entretanto, que os valores negativos de $u_{\mathrm{b}}$ não alteraram significativamente a forma da curva do ensaio e, consequentemente, os valores dos índices de compressibilidade ( $\mathrm{Cc}$ e $\mathrm{Cr}$ ).

A Tabela 4 mostra os índices de vazios iniciais $\left(\mathrm{e}_{\mathrm{o}}\right)$ e de compressibilidade (Cc e $\mathrm{Cr}$ ), além da tensão efetiva vertical de campo $\left(\sigma_{\text {vo }}\right)$ e de pré- adensamento $\left(\sigma^{\prime}{ }_{\mathrm{vm}}\right)$, calculada pelo método de Pacheco Silva. É curioso observar que, independentemente da qualidade da amostra e da geração de poropressão, não compatível com os limites propostos por diversos autores, os índices de compressibilidade normalizados, forneceram valores relativamente próximos; isto é, pode-se recomendar para uso prático os valores médios de $\mathrm{Cr} /\left(1+\mathrm{e}_{0}\right)$ e $\mathrm{Cc} /\left(1+\mathrm{e}_{0}\right)$ da ordem de 0,04 e 0,35 , respectivamente.

Tabela 4 - Parâmetros de compressibilidade e qualidade dos corpos de prova

\begin{tabular}{c|c|c|c|c|c|c|c|c|c|c}
\hline Ensaio & $\begin{array}{c}\sigma_{\mathrm{vo}} \\
(\mathrm{kPa})\end{array}$ & $\begin{array}{c}\sigma_{\mathrm{vm}} \\
(\mathrm{kPa})\end{array}$ & $\mathrm{OCR}$ & $\mathrm{e}_{\mathrm{o}}$ & $\mathrm{Cr}$ & $\mathrm{Cc}$ & $\Delta \mathrm{e} / \mathrm{e}_{0}$ & $\begin{array}{c}\text { Lunne et al } \\
(1997)\end{array}$ & $\begin{array}{c}\text { Coutinho et } \\
\text { al (1998) }\end{array}$ & $\begin{array}{c}\text { Andrade } \\
(2009)\end{array}$ \\
\hline $\begin{array}{c}\mathrm{CRS03} \\
5 \% / \mathrm{h}\end{array}$ & 29,50 & 58,00 & 2,0 & 4,28 & 0,15 & 1,85 & 0,06 & $\begin{array}{c}\text { Boa a A- } \\
\text { ceitável }\end{array}$ & Regular & $\begin{array}{c}\text { Boa a } \\
\text { Regular }\end{array}$ \\
\hline $\begin{array}{c}\mathrm{CRS04} \\
2 \% / \mathrm{h}\end{array}$ & 29,30 & 30,00 & 1,0 & 5,83 & 0,4 & 2,44 & 0,09 & Ruim & $\begin{array}{c}\text { Regular a } \\
\text { Ruim }\end{array}$ & Ruim \\
\hline $\begin{array}{c}\mathrm{CRS13} \\
2 \% / \mathrm{h}\end{array}$ & 29,20 & 28,00 & 1,0 & 6,38 & 0,38 & 2,69 & 0,12 & Ruim & Ruim & Ruim \\
\hline $\begin{array}{c}\mathrm{CRS14} \\
10 \% / \mathrm{h}\end{array}$ & 29,01 & 45,00 & 1,6 & 5,54 & 0,5 & 2,01 & 0,08 & Ruim & $\begin{array}{c}\text { Regular a } \\
\text { Ruim }\end{array}$ & $\begin{array}{c}\text { Boa a } \\
\text { Regular }\end{array}$ \\
\hline
\end{tabular}

Os resultados para os índices de compressibilidade $\mathrm{Cc}$ e $\mathrm{Cr}$ obtidos nos ensaios atuais do empreendimento apresentam valores próximos aos encontrados por Spannenberg (2003) na campanha experimental Rio -Polímeros II. Em ensaios SIC e CRS realizadas em amostras de boa qualidade e amolgadas, Spannenberg (2003) encontrou valores de Cc de 0,86 a 2,41 , e Cr de 0,16 a 0,28 .

O coeficiente de adensamento, calculado considerando-se o comportamento do solo como não linear
(Wissa et al, 1971), reduz com o aumento da tensão efetiva e tendeu a um valor constante da ordem de $1,5 \times 10^{-7} \mathrm{~m}^{2} / \mathrm{s}$. De fato, não há diferença significativa entre os comportamentos linear e não linear no cálculo de $\mathrm{c}_{\mathrm{v}}$, como observado por outros autores (Spannenberg, 2003; Vitor 2012). 


\section{COMPARAÇÃO ENTRE AS CAMPANHAS DE ENSAIOS}

\section{PERFIL GEOTÉCNICO}

Em 1997, a estratigrafia do terreno, nas proximidades da vertical V5, indicava $0,18 \mathrm{~m}$ de solo vegetal sobrejacente ao pacote argiloso superficial muito mole com 3,65 m de espessura, este último estando assente sobre uma camada de areia fina a média, com fragmentos de conchas, cinza e marrom escuro. Na região da vertical V6 o perfil indicava $0,15 \mathrm{~m}$ do solo vegetal e $3,85 \mathrm{~m}$ de argila muito mole, com as mesmas características.

$\mathrm{Na}$ fase de implantação do empreendimento comercial foi executado um aterro de $1,7 \mathrm{~m}$, incluindo $0,5 \mathrm{~m}$ de colchão drenante. Foi utilizada uma areia média limpa tanto para o aterro quanto para o colchão drenante. Segundo o contratante, nunca foi realizado nenhum trabalho de recolocação de aterro, após a sua construção.

Na presente campanha, após a retirada de todo material coletado nos Shelbies, verificou-se que a altura do aterro foi da ordem de 2,4 $\mathrm{m}$ e a espessura da camada argilosa variou de $1,76 \mathrm{~m}$, na vertical V5, a 0,34 m na vertical V6, conforme desenho esquemático indicado na Figura 13

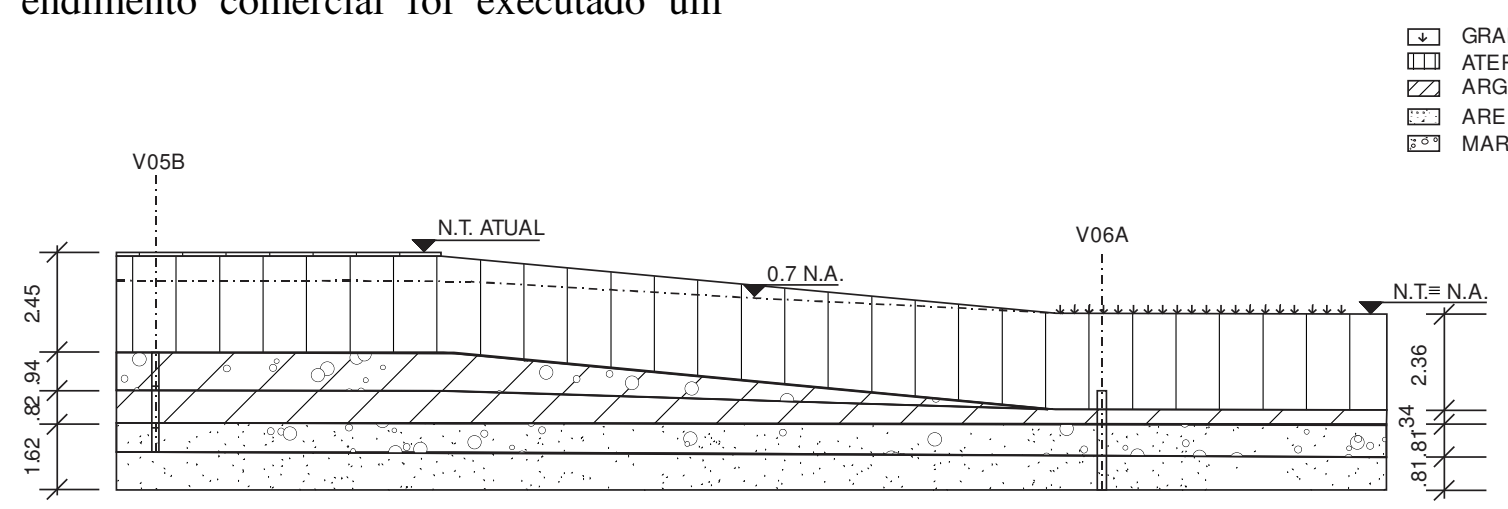

Figura 13 - Perfil geotécnico esquematizado a partir da abertura dos Shelbys no laboratório - 2012

Neste perfil, procurou-se basear na perfilagem obtida dos amostradores nas duas verticais, V5 e V6, assumindo que a camada arenosa subjacente ao pacote argiloso superficial não teve sua cota alterada com o carregamento do aterro. Acredita-se, portanto, que a camada drenante $(0,5 \mathrm{~m})$ tenha sido somada a espessura final $(1,7 \mathrm{~m})$ de projeto do aterro.

\section{PARÂMETROS GEOTÉCNICOS}

Para se estabelecer a equivalência entre amostras, houve necessidade de se definir uma relação entre as profundidades de retirada dos Shelbies antes da construção do aterro e na condição em 2012. Para tal, assumiu-se o topo da camada arenosa inferior como indeslocável e a partir deste referencial, foi possível equiparar as profundidades, como exemplifica a Tabela 5 para o caso da vertical V5. A partir desses dados foi possível correlacionar os ensaios realizados na vertical V5, antes e depois do aterro (Tabela 6) e definir 4 horizontes, denominados de A a D.

Quanto à caracterização (

Tabela 7), observam-se os efeitos do processo de adensamento em face da redução acentuada do índice de vazios (de 12 para 3,0 ), teor de umidade (de 418\% para 110\%) e limites de Atterberg entre campanhas. Já o peso específico apresentou um aumento pouco expressivo. 
Tabela 5 - Profundidades equivalentes - Vertical V5.

\begin{tabular}{c|c|c|c}
\hline \multicolumn{2}{c|}{$\begin{array}{c}\text { Antes do aterro (V5) } \\
\mathrm{H}_{\text {argila }}=3,65 \mathrm{~m} ; \\
\mathrm{H}_{\text {aterro }}=0 \mathrm{~m}\end{array}$} & \multicolumn{2}{c}{$\begin{array}{c}\text { Situação atual (V5B) } \\
\mathrm{H}_{\text {argila }}=1,76 \mathrm{~m} ;\end{array}$} \\
\hline $\mathrm{Z}$ argila $(\mathrm{m})$ & Z relativo $(\mathrm{m})$ & Z argila $(\mathrm{m})$ & Profundidade $(\mathrm{m})$ \\
\hline 0,58 & $16 \% \mathrm{H}$ & 0,28 & 2,64 \\
\hline 1,58 & $43 \% \mathrm{H}$ & 0,76 & 3,12 \\
\hline 2,58 & $70 \% \mathrm{H}$ & 1,23 & 3,59 \\
\hline 3,58 & $98 \% \mathrm{H}$ & 1,72 & 4,09 \\
\hline
\end{tabular}

Tabela 6 - Correlação entre ensaios das duas campanhas - vertical V5.

\begin{tabular}{c|c|c|c|c}
\hline \multirow{2}{*}{ Prof. } & Profundidade & Profundidade & \multicolumn{2}{|c}{ Ensaios de Adensamento } \\
\cline { 4 - 5 } & em 1997 $(\mathrm{m})$ & em 2012 $(\mathrm{m})$ & 1997 & 2012 \\
\hline A & $0,20-0,95$ & $2,45-2,80$ & SIC01 & CRS04, CRS13, CRS14 \\
\hline B & $1,20-1,95$ & $2,92-3,27$ & SIC02 & CRS03 \\
\hline C & $2,20-2,95$ & $3,39-3,74$ & SIC03 & - \\
\hline D & $3,20-3,95$ & $3,86-4,21$ & SIC04 & - \\
\hline
\end{tabular}

Tabela 7 - Ensaios de caracterização - valores médios.

\begin{tabular}{|c|c|c|c|c|c|c|c|}
\hline ANO & $\begin{array}{c}\gamma \text { nat } \\
\left(\mathrm{KN} / \mathrm{m}^{3}\right)\end{array}$ & $\mathrm{G}$ & $\mathrm{e}_{0}$ & $\begin{array}{c}\mathrm{w}_{\text {nat }} \\
(\%)\end{array}$ & $\begin{array}{c}\mathrm{LL} \\
(\%)\end{array}$ & $\begin{array}{c}\mathrm{LP} \\
(\%)\end{array}$ & $\begin{array}{c}\text { IP } \\
(\%)\end{array}$ \\
\hline 2012 & 13,6 & 2,6 & 3,0 & 115 & 135 & 41 & 90 \\
\hline 1997 & 11,1 & 2,6 & 12,0 & 460 & 595 & 154 & 440 \\
\hline
\end{tabular}

A Figura 14 compara as curvas de compressibilidade para as regiões A (mais superficial) e $\mathrm{B}$, onde são observados os seguintes pontos:

i) A tensão efetiva de pré-adensamento encontra-se dentro da ordem de grandeza compatível com a sobrecarga do aterro, que foi de cerca de $40 \mathrm{kN} / \mathrm{m}^{2}$. Houve, portanto aumento expressivo da tensão de pré-adensamento; os valores de OCR, obtidos para a segunda campanha, tendem a 1, como esperado.

ii) Redução em cerca de $50 \%$ do índice de compressibilidade $(\mathrm{Cc})$ para o horizonte $\mathrm{A}$ e cerca de $80 \%$ para o B. De fato, esperava-se que o valor de Cc não se alterasse, já que equivale à condição virgem. É possível que esta redução seja atribuída ao fato das amostras da presente campanha não terem sido classificadas como de boa qualidade. A má qualidade tende ao achatamento da curva e consequentemente à redução do índice de compressibilidade $\mathrm{Cc}$ e aumento do índice de compressibilidade Cr. Apesar das variações dos índices $\mathrm{Cc}$ e $\mathrm{Cr}$, a relação normalizada indica valores de $\mathrm{CR}$ $\left(\mathrm{Cc} /\left(1+\mathrm{e}_{0}\right)\right)$ razoavelmente constantes e uma leve redução de $\mathrm{RR}\left(\mathrm{Cr} /\left(1+\mathrm{e}_{0}\right)\right)$, como mostra a Figura 15.

Quanto ao coeficiente de adensamento (cv), houve, em ambas as profundidades, um aumento no valor médio da ordem de 10 vezes, atribuído a uma maior porcentagem de areia nos corpos de prova da campanha atual; na primeira campanha essa porcentagem era de $24 \%$. 


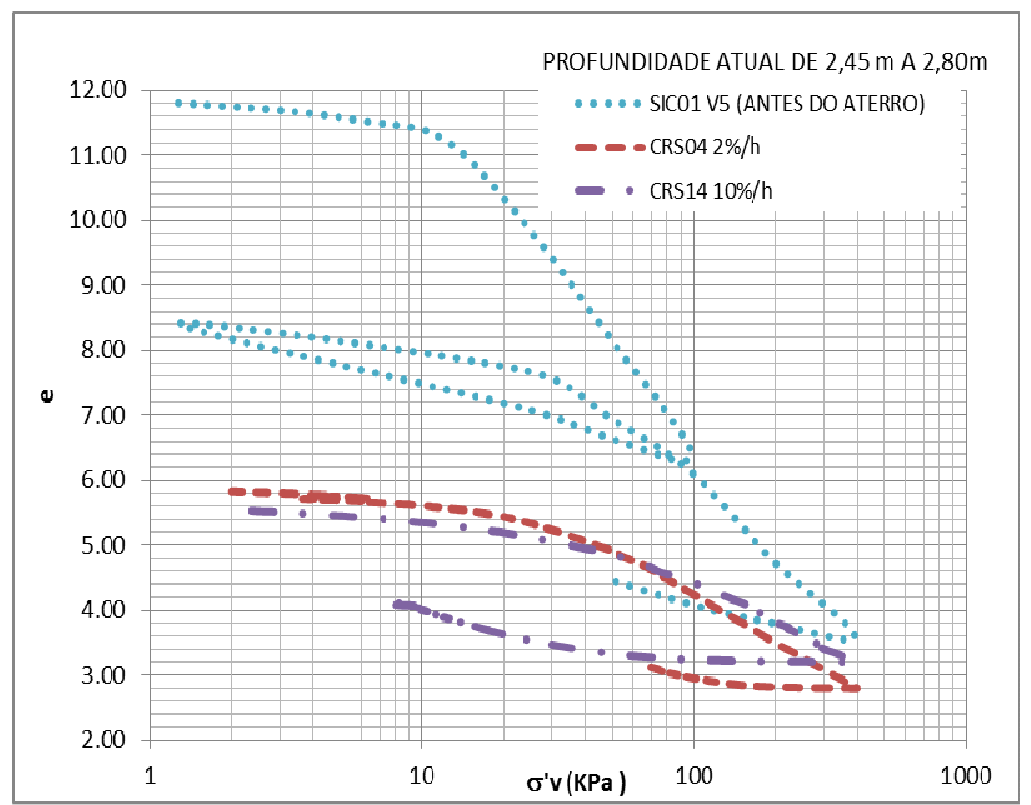

(a) Horizonte $\mathrm{A}$

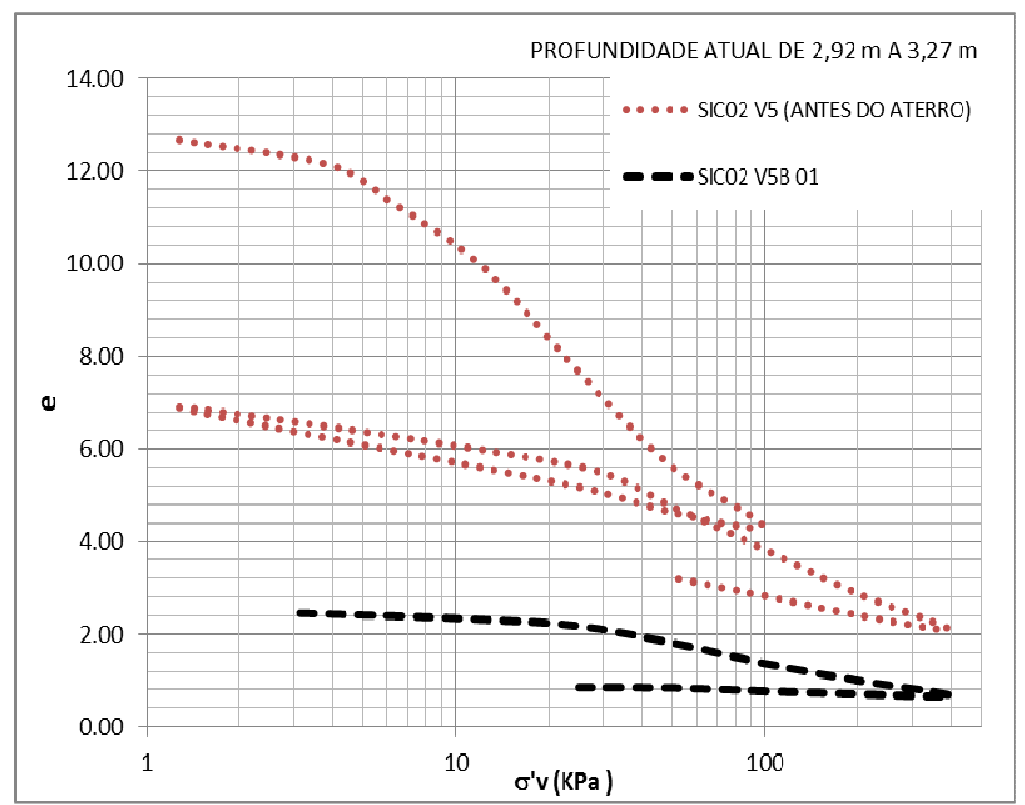

(b) Horizonte B

Figura 14 - Gráfico comparativo das curvas de compressibilidade
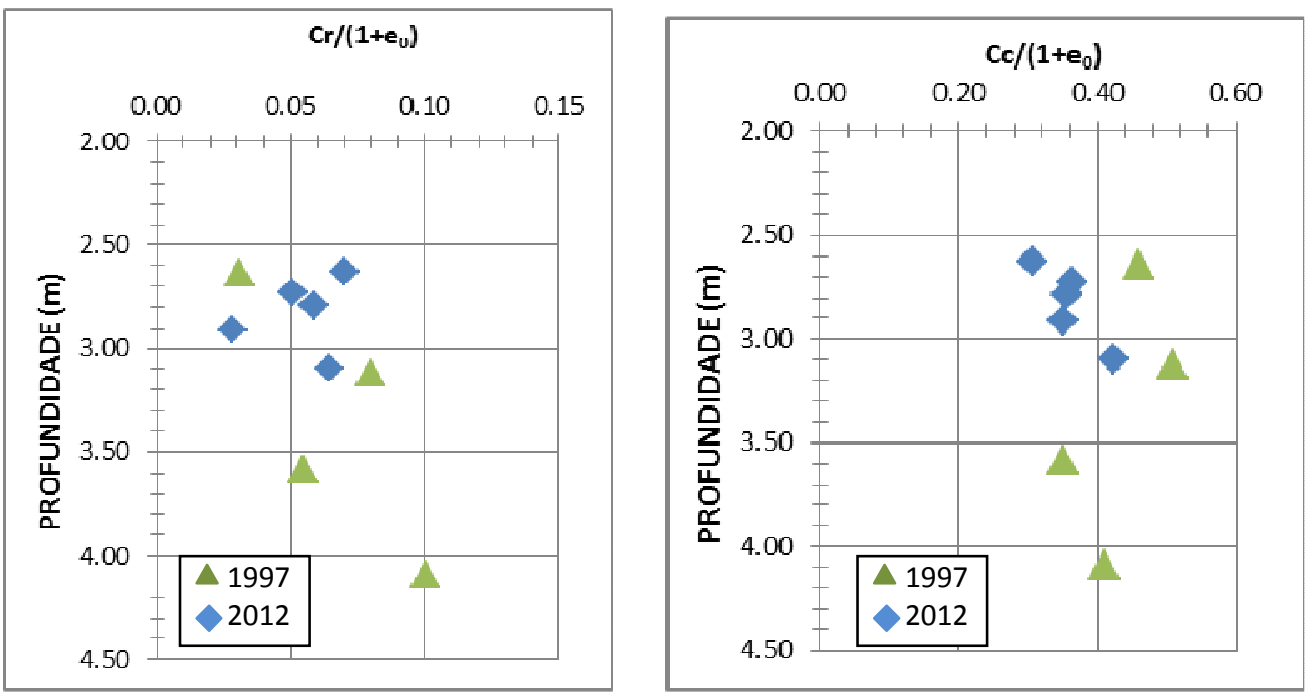

Figura 15 - Comparação entre índices de compressibilidade normalizados. 


\section{CONCLUSÕES}

$\mathrm{O}$ artigo apresentou resultados de ensaios de adensamento, SIC e CRS, em amostras retiradas 15 anos após a instalação de um aterro, bem como sua comparação com os resultados obtidos durante o projeto de um aterro num empreendimento situado na Av. Airton Senna, na Baixada de Jacarepaguá. As amostras foram coletadas junto às mesmas verticais onde foram coletadas as amostras anteriores, na primeira campanha de ensaios.

Comparou-se, para a campanha atual, o comportamento do solo argiloso muito compressível submetido aos ensaios SIC e CRS, este último para diferentes velocidades de deformação.

Foram também confrontadas as curvas de adensamento obtidas na campanha atual e aquelas obtidas por ocasião do projeto.

As principais conclusões observadas são listadas a seguir:

i) A qualidade dos corpos de prova obtidos das amostras da primeira campanha foi superior a dos corpos de prova obtidos na campanha atual, embora a sua retirada tenha sido realizada, supostamente, pela mesma equipe.

ii) Os critérios de classificação da qualidade dos corpos de prova foram considerados rigorosos quando comparados aos aspectos da curva de compressibilidade; a forma da curva sugere ser mais relevante do que a classificação em relação a $\Delta \mathrm{e} / \mathrm{e} 0$.

iii) Os ensaios de caracterização realizados em ambas as campanhas revelam a tendência de redução da porcentagem de finos com a profundidade. A porcentagem de areia é significativa, embora os demais índices físicos e o aspecto do solo indique um comportamento de solo argiloso. Cabe destacar que os ensaios da primeira campanha foram realizados pela Geotécnica em São Paulo e os da segunda foram realizados na UERJ, com equipamentos e equipes distintas.

iv) A formação do depósito em ambiente marinho foi verificada pela presença de conchas; v) Nas curvas granulométricas observouse redução significativa da \% argila entre as 2 campanhas

vi) Verificou-se, para fins práticos, que, a velocidade do ensaio CRS e a sua confiabilidade, quando da geração de valores negativos de $u_{b}$, pouco afetam os índices de compressibilidade, particularmente quando foram comparados os valores normalizados.

vii) Amostras amolgadas tendem a gerar valores de $\mathrm{u}_{\mathrm{b}} / \sigma_{\mathrm{v}}$ extremamente elevados no inicio do ensaio, sendo observada queda significativa após $100 \mathrm{kPa}$. Esse comportamento pode ser visto como um critério de análise da qualidade da amostra em ensaios CRS, independente dos critérios usualmente adotados para este fim.

\section{REFERÊNCIAS}

ANDRADE, M. E. 2009. Contribuição ao Estudo das Argilas Moles da Cidade de Santos. Dissertação de Mestrado. Rio de Janeiro, COPPE, UFRJ.

BALDEZ, B. S. 2013. Avaliação dos parâmetros de compressibilidade da camada de argila mole da Baixada de Jacarepaguá após longo período de sobrecarga de aterro. Dissertação de Mestrado. Rio de Janeiro. UERJ, RJ.

BARONI, M. 2010. Investigação Geotécnica em argilas orgânicas muito compressíveis em depósitos da Barra da Tijuca, Dissertação de Mestrado, COPPE/ UFRJ.

BEDESCHI, M. V. 2004. Recalques em Aterro Instrumentado Construído Sobre Depósito Muito Mole com Drenos Verticais na Barra da Tijuca, Rio de Janeiro. Dissertação de Mestrado. Rio de Janeiro: COPPE/UFRJ.

CARVALHO, S. R., ALMEIDA, M. D., \& MARTINS, I. S. 1993. Ensaios de adensamento com velocidade controlada: proposta de um método para definição da velocidade. Solos e Rochas, 16, 185-196. 
COUTINHO, R. Q. 2007. Characterization and Engineering Properties of Recife Soft Clays. 3, 2049 - 2100. (P. H. Tan, Ed.) London, Taylor and Francis - Balkema: Brazil.

COUTINHO, R. Q., OLIVEIRA, J, \& OLIVEIRA, A. 1998. Estudo Quantitativo da Qualidade de Amostras de Argilas Moles Brasileiras - Recife e Rio de Janeiro. COBRAMSEG, 2, 927-936.

LUNNE, T., BERRE, T., \& STRANDVIK, S. 1997. Sample Disturbance Effects in Soft Low Plastic Norwegian Clay. Recent Developments in Soil and Pavement Mechanics. 81-102. Rotterdam: Almeida.

SANDRONI, S. S. (1980). Depósitos Moles: Formação, Tipos e Análise de Estabilidade de Aterros sob Condições Não Drenadas. Relatório AT 20/80. Rio de Janeiro

SANDRONI, S. S. 2012. Comunicação pessoal.

SPANNENBERG, M. G. 2003. Caracterização geotécnica de um depósito de argila mole da Baixada Fluminense. Dissertação de Mestrado . Pontifícia Universidade Católica da Rio de Janeiro.

SPOTTI, A. P. 2006. Aterro Estaqueado Reforçado Instrumentado Sobre Solo Mole. Tese de Doutorado . Rio de Janeiro, COPPE/UFRJ.

SPOTTI, A. P. 2000. Monitoramento de Aterro Sobre Argila Orgânica Mole com Drenos Verticais. Dissertação de Mestrado, Rio de Janeiro, COPPE/UFRJ.

VITOR, K. A. 2012. Verificação do desempenho do ensaio de adensamento CRS comparado ao SIC. Dissertação de Mestrado . Rio de Janeiro, RJ, Universidade do Estado do Rio de Janeiro.

WISSA, A. E.-A. 1971. Consolidation at constant rate of strain. Journal of the soil mechanics and foundations division, (pp. 1393-1413 